\title{
AUTOCONCEPTO, AUTOEFICACIA Y PERCEPCIÓN DE CALIDAD DE VIDA EN NIÑOS Y NIÑAS CON Y SIN DÉFICIT DE PROCESAMIENTO SENSORIAL
}

\author{
SELF-CONCEPT, SELF-EFFICACY AND QUALITY OF LIFE IN BOYS AND GIRLS WITH \\ AND WITHOUT SENSORY PROCESSING DEFICIT
}

\section{María Carolina Maldonado Gelder', Zoraide María Lugli Rivero²}

\begin{abstract}
RESUMEN
Objetivo. El Autoconcepto, la autoeficacia y la calidad de vida son variables que pueden verse afectadas en los niños/as por la presencia de un déficit de procesamiento sensorial (DPS). Este déficit se caracteriza por fallas en el procesamiento de las sensaciones que pueden ocasionar dificultades en el desempeño cotidiano. La presente investigación tuvo por objetivo determinar si existe diferencia en el autoconcepto, la autoeficacia y la calidad de vida de niños/as venezolanos/as entre 7 y 13 años con y sin DPS.

Metodología. El presente es un estudio transversal, con diseño estadístico no experimental de comparación de medias mediante el estadístico t de Student. La muestra estuvo constituida por 98 niños/as de 7 a 13 años, de los cuales 38 (38,8\%) estaban diagnosticados con déficit de procesamiento sensorial y $60(61,2 \%)$ sin déficit.

Resultados. Se evidenció que existen diferencias significativas en la autoeficacia académica, donde los niños/as con DPS mostraron puntajes medios menores; asícomo en el autoconcepto físico, donde los puntajes fueron menores en niños/as sin déficit. No se encontraron diferencias significativas en la calidad de vida de las dos muestras. Esta investigación permitió abrir una línea de investigación en el área de variables psicológicas que han sido poco estudiadas en niños/as con DPS, demostrando que esta condición impacta principalmente su percepción de autoeficacia.
\end{abstract}

\section{PALABRAS CLAVE}

Niño, Funciones Sensoriales, Autoconcepto, Autoeficacia, Calidad de Vida (DeCS)

\begin{abstract}
SUMMARY
Objective. Self-concept, self-efficacy and quality of life are variables that can be affected in children by the presence of a sensory processing deficit (DPS). This deficit is characterized by failures in the processing of sensations that can cause difficulties in daily performance. The objective of this study is to determine whether or not there is a significant difference in the self-concept, self-efficacy and quality of life of Venezuelan children between 7 and 13 years old with and without Sensory Processing Difficulties (SPD).
\end{abstract}

1 Terapeuta Ocupacional - Universidad Nacional de Colombia (UN) Magister en Psicología. Universidad Simón Bolívar (USB) E - mail: carolinamaldonadogelder@gmail.com. Teléfono: (57) 3005532185

2 Psicólogo - Universidad Central de Venezuela (UCV) Magister en Psicología - Universidad Simón Bolívar (USB) Doctorado en Psicología en Curso - Universidad Central de Venezuela (UCV) E - mail: zlugli@gmail.com Teléfono: (58) 4166307585

Institución de afiliación principal: Universidad Simón Bolívar. Caracas, Venezuela.

ORCID: https://orcid.org/0000-0001-9462-2529 


\begin{abstract}
Methodology. This is a cross-sectional study, with a non-experimental statistical design of comparison of means using the student's t-statistic. The sample consisted of 98 children from 7 to 13 years old, of whom 38 (38.8\%) were diagnosed with sensory integration deficit and 60 (61.2\%) without deficit.

Results. There was evidence of significant differences in academic self-efficacy, where children with SPD showed lower mean scores; as well as in physical self-concept, where children without SPD attained lower scores. No significant differences were found in the quality of life of the samples. This study contributes to a line research in psychological variables that have been little studied in children with sensorial integration deficit, demonstrating that this condition mainly impacts their perception of self-efficacy.
\end{abstract}

\title{
KEYWORDS
}

Child, Sensory functions, Self-concept, Self-efficacy, Quality of Life (MeSH)

Recibido: 01/09/2020

Aceptado: 18/11/2020

\section{INTRODUCCIÓN}

Todas las personas utilizan diferentes estrategias que los ayudan a relajarse, activarse y organizarse para responder adecuadamente a las diferentes situaciones de la vida. Por ejemplo, algunos sienten que un baño con agua caliente los relaja, otros escuchan música para concentrarse o por el contrario necesitan silencio para poder prestar atención; en momentos necesitan estar con personas o ir a una fiesta, pero en otros no quieren que nadie se les acerque y prefieren estar solos.

Estas preferencias están determinadas por la manera como se procesa e integra la información sensorial proveniente del cuerpo y del ambiente, lo cual Ayres (1998) definió como Integración Sensorial, haciendo referencia a "la organización de las sensaciones para su uso" (p. 13), convirtiendo dicha estimulación en percepción con la finalidad de responder a partir de ella a las demandas cotidianas.

El cerebro debe aprender a organizar y procesar los estímulos sensoriales y usarlos para responder adaptativamente en una situación particular, lo que requiere de innumerables procesos neurológicos que permiten localizar, clasificar y ordenar las sensaciones, igual que un agente de tráfico organiza el paso de vehículos en un cruce (Ayres, 1998). Cuando las sensaciones de nuestro cuerpo son integradas y organizadas eficazmente, podemos tener una percepción precisa de nuestro mundo, lo que a su vez nos permite emitir comportamientos regulados y adquirir nuevos aprendizajes. De lo contrario, nos enfrentamos a un gran número de estímulos a los que no podemos responder de manera eficaz y que pueden dificultar nuestro desempeño cotidiano, alterar nuestro comportamiento e impactar el componente emocional, con relación a la percepción de nosotros mismos y de nuestras capacidades (Smith, 2006).

En el caso de los niños/as, Ayres (1998) plantea que cuando el flujo de sensaciones es caótico, su vida es parecida "al tráfico en una hora pico" (p. 14). Cuando esto sucede, se presentan dificultades al participar en las actividades cotidianas (alimentación, vestido, higiene, actividades escolares, sociales, lúdicas y deportivas) ya que el/la niño/a ve limitada su capacidad para filtrar la información que llega de sus sentidos, por lo que no sabe a qué estímulos responder y qué estímulos ignorar, mostrándose desorganizado y fuera de sincronía con su medio ambiente; cuando esto sucede, se puede afirmar que existe un Déficit de Procesamiento Sensorial (DPS) (Fisher, Murray \& Bundy 1991).

Debido a las dificultades para procesar adecuadamente la información sensorial del ambiente y de su propio cuerpo, los niños/as con DPS se enfrentan constantemente al fracaso, lo que puede generar sentimientos de frustración, incompetencia, baja confianza en sí mismos y en sus capacidades (Parham \& Mailloux, 2001).

Para algunos autores, la baja auto-estima y poca confianza en sí mismo son efectos indirectos del DPS (Ayres, 1998; Parham \& Mailloux, 2001; Schaaf \& Smith, S., 2006); el niño hace un esfuerzo constante por completar las tareas, lo que hace comprensible su usual frustración.

La frustración aumenta cuando observa que sus pares completan las tareas sin esfuerzo y a la larga la frustración crónica provoca el arraigo de sentimientos de incompetencia, baja 
auto-confianza y auto-eficacia, desarrollando sentimientos de minusvalía, limitando aún más las posibilidades de que el niño se atreva a participar en actividades que le brinden experiencias integradoras (Parham \& Mailloux, 2001, p. 356).

Este impacto que puede tener el DPS sobre el mundo emocional del niño/a, se puede ver en estudios como el de Cairney, Hay, Faught, Wade, Corna \& Flouris (2005), quienes estudiaron la autoeficacia para la actividad física y la participación en actividades de juego en 44 niños/as canadienses de 4 to a $8 v o$ grado, con desorden del desarrollo de la coordinación y encontraron que estos niños/as tienen menos probabilidades de ser físicamente activos y que la autoeficacia generalizada puede dar cuenta de una proporción considerable de esta relación, ya que los/as niños/as con baja autoeficacia generalizada tienden a participar menos en actividades físicas y en el juego que los/as niños/as que poseen mayor autoeficacia generalizada.

Así mismo, Plata \& Garbiñe, (2009), plantean que los/ las niños/as con DPS, específicamente con desorden del desarrollo de la coordinación, presentan dificultades que los hacen poco populares, teniendo que soportar incluso, en algunas ocasiones, acoso y burla por parte de sus compañeros. Estos niños/as ven restringida su participación en situaciones de la vida cotidiana, lo cual posiblemente lo pone en riesgo de experimentar aislamiento social, victimización y rechazo por parte de sus pares (Poulsen \& Ziviani, 2004).

Otros estudios relacionados con el DPS (Parhan \& MaiIlloux, 2001; Bundy, Lane \& Murray 2002; Cairney, et al. 2005) abordan de manera indirecta el componente emocional, resaltando que estos/as niños/as presentan una condición que si no es abordada adecuadamente, los lleva paulatinamente a mostrar niveles crecientes de dificultad y esfuerzo para lograr rendimientos acordes a las demandas ambientales, especialmente escolares, con una consecuente disminución en el concepto positivo de sí mismos/as y en su confianza personal, aspectos fundamentales para enfrentar desafíos futuros y para experimentar bienestar y sentirse felices. En otros casos, la problemática podría agudizarse a nivel de relaciones interpersonales y participación social, de estabilidad emocional o de limitación de las elecciones ocupacionales, es decir, del tipo de actividades escolares, recreativas, sociales, deportivas, culturales, entre otras, que ese/a niño/a podrá elegir como ejes de su vida (Colombo, 2003), lo que en definitiva va a tener un impacto importante en su calidad de vida.

Así lo plantean Caçola \& Killian (2018), quienes evaluaron a niños/as de 6 a 12 años con desorden del desarrollo de la coordinación, mediante el reporte de los padres con los instrumentos PedsQL y KIDSCREEN que miden calidad de vida relacionada con la salud en población infantil. Los resultados mostraron puntuaciones bajas en los dos instrumentos, lo que indica que el desorden del desarrollo de la coordinación probablemente impacta negativamente en la percepción de calidad de vida de los/as niños/as.

De la misma manera, Karras, Morin, Gill, Izadi-Najafabadi \& Zwicker (2019), compararon la calidad de vida relacionada con la salud en niños/as con y sin desorden del desarrollo de la coordinación en una edad media de 9,8 años, encontrando que los niños/as con desorden del desarrollo de la coordinación y sus padres informaban una calidad de vida relacionada con la salud mucho menor. El estudio concluyó por un lado que estos/as niños/as tienen mayores alteraciones emocionales y de comportamiento y por otro que las dificultades en el desempeño motor predicen la calidad de vida relacionada con la salud.

La literatura en el área da cuenta de la manera como el componente emocional se puede ver afectado negativamente cuando los/as niños/as se enfrentan a constantes dificultades para participar en su ambiente, ya sea escolar, familiar o social, lo que limita el desarrollo de habilidades y competencias fundamentales para el bienestar y la calidad de vida en general (Pfeiffer, Coster, Snethen, Derstine, Piller \& Tucker, 2017).

Si bien la evidencia científica en aspectos como el desempeño ocupacional, la evaluación e intervención de estos/as niños/as, entre otros, es muy amplia, es necesario fortalecer la evidencia relacionada con el impacto que tiene el DPS sobre el componente emocional y la calidad de vida de los/as niños/as, teniendo en cuenta que, como plantean Bundy et al., (2002), debido a las diferentes habilidades que se ven afectadas en el DPS, el/ la niño/a puede empezar a limitar su participación en ocupaciones que son propias de la niñez y que favorecen el desarrollo de habilidades y destrezas necesarias para interactuar con su ambiente y para construir una percepción positiva de sí mismo/a. Es por esto, por lo 
que, como plantea la American Occupational Therapy Association (2014), aspectos relacionados con la regulación emocional como la autoestima en niños/as con DPS, deben ser áreas de investigación prioritaria.

De igual forma, es de vital relevancia que las intervenciones de Terapia Ocupacional en niños/as con DPS estén encaminadas hacia el logro de mayores niveles de bienestar para el/la niño/a y su familia, mediante estrategias relacionadas con el establecimiento de metas que cumplan con el criterio de "Desafío Justo" en los diferentes ambientes, comprensión de expectativas reales en el/la niño/a, padres y maestros, estrategias o modificaciones ambientales que apoyen el desempeño y en general un abordaje no solo desde la clínica sino además en los ambientes cotidianos, ya que es a través de la participación en estos ambientes, que el/la niño/a construye su identidad, moldea el concepto de sí mismo/a, fortalece la creencia en sus habilidades y alimenta la percepción sobre su calidad de vida.

\section{MÉTODO}

\section{Muestra}

Se aplicó un muestreo no probabilístico e intencional, que permitió seleccionar la muestra definitiva con las características requeridas para la investigación (Kumar, 2011). La muestra constituida por 98 niños/as residentes y escolarizados en la ciudad de Caracas (Venezuela), distribuidos en dos grupos, un grupo de 38 niños/ as $(38,4 \%)$ con DPS y un grupo de 6 o niños/as $(60,6 \%)$ sin déficit, residentes y escolarizados en la ciudad de Caracas. Los representantes de todos los niños/as aceptaron voluntariamente que sus hijos participaran en la investigación.

La muestra de niños/as con DPS fue recogida en diferentes centros de terapia de la ciudad de Caracas. Para ello se seleccionaron aquellos niños/as con un puntaje entre 38 y 141 en el perfil sensorial (Dunn, 1999) contestado por los padres y que a su vez hubiesen sido diagnosticados por sus especialistas con DPS, sin ningún otro diagnóstico comórbido. La muestra de niños/ as sin DPS fue seleccionada de manera aleatoria en dos colegios de la ciudad de Caracas, uno público y uno privado, cuyas respuestas al perfil sensorial (Dunn, 1999) estuviera entre 155 y 190 (funcionamiento típico) y sus padres reportaran no tener ningún diagnóstico de trastorno del neurodesarrollo.

La muestra definitiva estuvo compuesta por un total de 98 niños/as en edades comprendidas entre 7 y 13 años, cuyas características se describen en la tabla 1 y 2.

TABLA 1 CARACTERÍSTICAS DE LA MUESTRA GENERAL

\begin{tabular}{lll}
\hline MUESTRA GENERAL & & \\
\hline Sexo & Frecuencia & Porcentaje \\
Femenino & & \\
Masculino & 41 & 41.4 \\
Edad & 57 & 57.6 \\
7 & & \\
8 & 5 & 5.1 \\
9 & 16 & 16.2 \\
10 & 20 & 20.2 \\
11 & 31 & 21.3 \\
12 & 14 & 14.1 \\
13 & 8 & 8.1 \\
Tipo de escuela & 4 & 4 \\
Privada & & \\
Pública & 66 & 66.7 \\
Escolaridad (grado) & & 32.3 \\
1 & 32 & \\
2 & 3 & 3 \\
3 & 11 & 11.2 \\
4 & 23 & 23.2 \\
En la tabla 2 se presentan las características de cada gru- \\
po en función de la presencia o no de D 69. \\
6 & 18 & 18.2 \\
\hline
\end{tabular}

\section{Tipo y Diseño de Investigación}

La presente investigación fue de tipo no experimental de comparación de grupos ya que no se manipularon las variables del estudio con el fin de establecer control experimental (Ato \& Vallejo, 2015). También es una investigación transversal, ya que la recolección de los datos se llevó a cabo en un solo momento, en un tiempo único, con el objeto de describir las variables y comparar su comportamiento en cada uno de los grupos en 
AUTOCONCEPTO, AUTOEFICACIA Y PERCEPCIÓN DE CALIDAD DE VIDA EN NIÑOS Y NIÑAS CON Y SIN DÉFICIT DE PROCESAMIENTO SENSORIAL

TABLA 2 CARACTERÍSTICAS DE CADA GRUPO EN FUNCIÓN DE LA PRESENCIA O NO DE DPS.

\begin{tabular}{|c|c|c|c|c|c|}
\hline \multicolumn{3}{|c|}{ NIÑOS/AS CON SPS $(n=60)$} & \multicolumn{3}{|c|}{ NIÑOS/AS SIN SPS(n=38) } \\
\hline Edad & & & Edad & & \\
\hline \multicolumn{3}{|c|}{ Media $=10.3$} & \multicolumn{3}{|c|}{ Media $=8.8$} \\
\hline & Frecuencia & Porcentaje & & Frecuencia & Porcentaje \\
\hline \multicolumn{3}{|l|}{ Sexo } & \multicolumn{3}{|l|}{ Sexo } \\
\hline Femenino & 30 & 50 & Femenino & 11 & 28.9 \\
\hline Masculino & 30 & 50 & Masculino & 27 & 71.1 \\
\hline \multicolumn{3}{|c|}{ Escolaridad (grado) } & \multicolumn{3}{|c|}{ Escolaridad (grado) } \\
\hline 1 & 0 & 0 & 1 & 3 & 7.9 \\
\hline 2 & 0 & 0 & 2 & 11 & 28.9 \\
\hline 3 & 10 & 16.7 & 3 & 13 & 34.2 \\
\hline 4 & 31 & 51.7 & 4 & 6 & 15.8 \\
\hline 5 & 1 & 1.7 & 5 & 5 & 13.2 \\
\hline 6 & 18 & 30 & 6 & 0 & 0 \\
\hline
\end{tabular}

un momento dado (Hernández, Fernández \& Baptista, 2014). Dentro de este tipo de diseño puede considerarse como una investigación descriptiva ya que busca describir el comportamiento de las variables en cada uno de los grupos y observar si existen diferencias.

Por tratarse de una investigación no experimental y por los objetivos planteados, se utilizó un diseño estadístico no experimental, de comparación de medias, el cual permitió comparar el comportamiento de las variables en los grupos de niños/as con y sin DPS.

\section{INSTRUMENTOS}

\section{Breve Perfil Sensorial (Winnie Dunn, 1999)}

Realizado por Dunn (1999). Es una lista de chequeo que fue aplicada a padres para identificar las respuestas de los/as niños/as entre 5 y 10 años ante diferentes experiencias sensoriales, permitiendo identificar la presencia o no del déficit. Se utilizó el formato corto propuesto por la autora como ideal para protocolos de investigación (1999). Consta de 38 ítems distribuidos en 7 dimensiones: Sensibilidad Táctil, Sensibilidad Gustativa/Olfatoria, Sensibilidad al Movimiento, Poco
Sensible/Busca Sensación, Filtro Auditivo, Baja Energía/ Débil, Sensibilidad Visual/Auditiva. Los padres deben responder a cada uno de los ítems según las etiquetas siempre 1 «Siempre»; 2 «Frecuentemente»; 3 «A veces»; 4 «Casi Nunca»; y 5 «Nunca». El puntaje total considerado como funcionamiento típico es de 155 a 190, los puntajes que estén por debajo denotan una alteración del procesamiento sensorial. Los valores de Alfa de Cronbach de este perfil varían de .47 a .91, sugiriendo una confiabilidad de consistencia interna moderada a alta en las distintas dimensiones. Para la validez de constructo, el manual presenta una estructura factorial realizada en una muestra de 1032 niños/as (no especifican características) de 7 factores claramente diferenciados: sensibilidad táctil, sensibilidad al gusto/olfato, sensibilidad al movimiento, hiposensible/búsqueda de sensación, filtro auditivo, energía baja/débil, sensibilidad visual/auditiva. Como indicadores de validez interna señalan intercorrelaciones dimensión-factor de moderadas a bajas.

\section{Escala de Evaluación de Autoconcepto para Niños de Piers-Harris (Piers \& Harris 1969).}

La escala inicial, dirigida a niños/as entre 7 y 12 años, está compuesta por 80 ítems, que miden las siguientes dimensiones: Autoconcepto Conductual, 
Autoconcepto Intelectual, Autoconcepto Físico, Falta de Ansiedad, Popularidad y Felicidad-Satisfacción. Los ítems son puntuados con una escala dicotómica (SI $\mathrm{NO})$ que permite obtener información sobre la percepción que el niño/a tiene de sí mismo/a y de la manera en que autoevalúa diferentes aspectos relacionados con su comportamiento. Un alto puntaje en la escala sugiere una autoevaluación positiva sobre el concepto de sí mismo, mientras un puntaje bajo, sugiere una autoevaluación negativa del mismo.

La Escala fue adaptada y validada en una muestra de 26o niños/as venezolanos entre 7 y 12 años como fase inicial del presente trabajo. La validez de constructo se obtuvo a través del análisis factorial exploratorio con rotación varimax, arrojando una estructura de seis factores a partir del autovalor mayor a 1: Autoconcepto Conductual que explica el $11,480 \%$ de la varianza total con un Alfa de Cronbach de 0.81, Autoconcepto Social con 5,897\% de varianza explicada y un alfa de 0.46, Autoconcepto Emocional con 4,019\% de varianza explicada y un alfa de o-75, Felicidad - Satisfacción con 3,476\% de varianza y un alfa de 0.74 , Autoconcepto Físico con 3,025\% de varianza y un alfa de 0.68 y Autoconcepto General con $2,754 \%$ de varianza y un alfa de 0.65 . El Alpha de Cronbach obtenido para la totalidad de la prueba es de 0.867 , lo cual demuestra un buen nivel de fiabilidad.

\section{Escala de Autoeficacia para Niños (Bandura, 1990), adaptada y validada en España por Carrasco \& Del Barrio (2002):}

La escala está compuesta por 35 ítems seleccionados de un grupo de ocho dimensiones procedentes de un gran grupo de escalas de autoeficacia multidimensional de Bandura (Bandura, 1990). Su objetivo es explorar la percepción de autoeficacia en niños/as de 8 a 15 años, a través de tres dimensiones: Autoeficacia Académica, Autoeficacia Social y Autoeficacia Autorregulatoria. El formato de respuesta está graduado de cero a cinco y acompañado en cada uno de los niveles por etiquetas de distinta intensidad de eficacia con el fin de facilitar la comprensión de la graduación numérica ( 1 «Fatal»; 2 «no muy bien»; 3 «bien»; 4 y 5 «fenomenal»). Un alto puntaje en la escala sugiere una autoevaluación positiva sobre sus capacidades, mientras un puntaje bajo, sugiere una autoevaluación negativa de las mismas.
La Escala fue adaptada y validada en una muestra de 347 niños/as venezolanos entre 7 y 12 como fase inicial del presente trabajo. La validez de constructo se obtuvo a través del análisis factorial exploratorio con rotación varimax, arrojando una estructura de dos factores: Autoeficacia Académica que explica el 21,96\% de la varianza total con un alfa de Cronbach de 0.87 y Autoeficacia Lúdico Social con una varianza explicada de $28,77 \%$ y un alfa de 0.76. El Alpha de Cronbach obtenido para la totalidad de la prueba es de o.88, lo cual demuestra un buen nivel de fiabilidad.

\section{Cuestionario KIDSCREEN-52 para medir Calidad de Vida Relacionada con la Salud, adaptada y validada en población venezolana por Guzmán (2012)}

El Kidscreen fue creado por el Grupo KIDSCREEN, (Ravens-Sieberer, Erhardt, Wille, Wetzel, Nickel \& Bullinger, 2006). Es el primer instrumento de calidad de vida relacionada con la salud para población infantil y adolescente entre 8 y 18 años, desarrollado simultáneamente en trece países europeos (Alemania, Austria, España, Francia, Grecia, Países Bajos, Hungría, Irlanda, República Checa, Polonia, Reino Unido, Suecia y Suiza). Está constituida por 52 preguntas distribuidas en 10 dimensiones respondidas en escala tipo likert de intensidad y frecuencia, cada una con cinco categorías de respuesta, las puntuaciones directas de cada dimensión se obtienen a partir de una media de ítems (rango 1 - 5), cada dimensión se transforma a una escala de o a 100 puntos, donde una mayor puntuación representa una mayor CVRS (Guzmán, 2012). La Escala fue traducida y validada en población española en el año 2004 y posteriormente se llevó a cabo la adaptación y estudio de validez y fiabilidad en una muestra de 360 niños/ as y adolescentes entre 8 y 18 años por Guzmán (2012). La validez de constructo se obtuvo utilizando un análisis factorial exploratorio con rotación varimax, arrojando como resultado una estructura factorial de diez dimensiones que explican el $55.8 \%$ de la varianza total: Bienestar Físico, Bienestar Psicológico, Estado de Ánimo, Autopercepción, Autonomía, Relación con los Padres, Recursos Económicos, Amigos y Apoyo Social, Entorno Escolar y Aceptación Social. La fiabilidad valorada a través del coeficiente alfa de Cronbach oscila entre 0,73 a o,85 en las diferentes dimensiones. 


\section{Procedimiento}

La presente investigación se llevó a cabo en tres fases que se describen a continuación:

\section{Fase 1. Revisión de literatura y delimitación del problema de investigación}

Se realizó una exhaustiva revisión de la bibliografía y se realizaron entrevistas a expertos en el tema, con el fin de delimitar el problema de investigación, establecer con claridad los objetivos de investigación y enriquecer el marco teórico que sustenta el estudio.

Durante esta misma fase se determinó el diseño de investigación más idóneo para dar respuesta a los objetivos planteados y se procedió a la búsqueda y escogencia de los instrumentos de evaluación para medir las variables propuestas.

\section{Fase 2. Validez y Fiabilidad de los Instrumentos de Medida (cuestionarios)}

En esta fase se llevó a cabo el estudio de validez y fiabilidad en una muestra de niños/as venezolanos de los instrumentos que lo requerían: Escala de Evaluación de Autoconcepto para Niños de Piers-Harris (Piers \&Harris, 1969) y Escala de Autoeficacia para Niños (Bandura, 1990), adaptada y validada en España por Carrasco \& Del Barrio (2002). Para cada instrumento se realizó un análisis factorial exploratorio para determinar la validez de constructo y análisis de consistencia interna para el índice de confiabilidad. Los resultados de esta fase están descritos en el apartado de instrumentos del presente artículo.

\section{Fase 3. Medición de las Variables de Estudio en la Muestra Definitiva}

La propuesta de investigación fue revisada y aprobada por el Decanato de estudios de Postgrado de la Universidad Simón Bolívar quien se encarga de valorar los aspectos teóricos, metodológicos y éticos de los estudios. Se respetaron las normas éticas explicitas en la declaración de Helsinki (2013). Se solicitó por escrito a las direcciones de las escuelas seleccionadas y de los centros de terapia el permiso para notificar a los padres sobre los objetivos de la investigación y la participación de sus hijos/as. Los padres que aceptaron que sus hijos/ as participaran en la investigación firmaron un consentimiento informado que incluyó una breve explicación del estudio y en qué consistía la participación (contestar cuestionarios), así como dar su permiso para la divulgación y publicación de los resultados en eventos y revistas científicas.

Posterior a lo señalado en el párrafo anterior, se aplicó el paquete de instrumentos a niños/as regulares de dos instituciones educativas de la ciudad de Caracas y posteriormente se estableció un cronograma para llevar a cabo la aplicación de los instrumentos a niños con DPS, los cuales asistían a diferentes centros de terapia ubicados en la ciudad de Caracas.

Finalmente se creó la base de datos que permitió el cálculo de los respectivos análisis estadísticos con el paquete SPSS, versión 18.0.

\section{Análisis de Datos}

En virtud que se tienen dos grupos se utilizó el estadístico t de Student de muestras independientes para determinar si existían diferencias significativas entre ellos en cada una de las dimensiones de las variables, autoeficacia, autoconcepto y calidad de vida, presentando estadísticos descriptivos para analizar las diferencias encontradas.

Se tomó un nivel de significancia del 0,05. Previamente se evaluó el supuesto de homogeneidad de varianzas utilizando la prueba de Levene, la cual indicó no ser significativa para todas las dimensiones de las variables autoconcepto y autoeficacia, cumpliendo con el criterio de homocedasticidad; para algunas dimensiones de la variable calidad de vida (bienestar psicológico, estado de ánimo, autonomía, amigos y apoyo social, entorno escolar y aceptación social) se obtuvo heterogeneidad de varianza entre los grupos, por lo que se tomó el valor de t de Student corregido por los grados de libertad, usado cuando no se asume igualdad de varianza.

\section{Resultados}

Para comparar cada una de las dimensiones de las variables autoconcepto, autoeficacia y calidad de vida en 
función de la presencia o ausencia del DPS, se compararon las medias de cada grupo mediante la prueba de diferencia t de Student de muestras independientes.

A partir del análisis de los datos se puede observar que, con relación al autoconcepto, los/as niños/as con y sin DPS no muestran diferencias significativas, a excepción del autoconcepto físico en el que los/as niños/as con Déficit de Integración Sensorial mostraron tener una mejor percepción de sí mismo con relación a sus características físicas (Tabla 3).

En la tabla 4 se observa que existen diferencias significativas entre ambos grupos solamente en autoeficacia académica, siendo mayor los puntajes en los niños/as sin DPS.

Finalmente, al analizar la variable calidad de vida se puede observar en la Tabla 5 que no existen diferencias significativas entre los dos grupos en ninguna de sus dimensiones.

\section{Discusión}

Cuando revisamos la literatura referente al DPS, encontramos que los niños/as que tienen alteraciones en el procesamiento de sus sensaciones, presentan dificultades en el desempeño de las actividades cotidianas, en la coordinación motora, atención, aprendizaje, y en su desarrollo emocional y social (Serrano, 2019). Estos/ as niños/as se enfrentan a constantes dificultades para participar en su ambiente, ya sea escolar, familiar o social, lo que limita el desarrollo de habilidades y competencias fundamentales para el bienestar y la calidad de vida en general, es por ello por lo que el objetivo general de esta investigación estuvo dirigido a determinar si existen diferencias en el autoconcepto, la autoeficacia y la calidad de vida de niños/as con y sin DPS.

Con respecto al Autoconcepto, los resultados demostraron que tanto los/as niños/as con DPs como los/as niños/as sin déficit, poseen puntajes moderados a altos en esta variable, lo que indica que los/as niños/as de ambos grupos poseen un concepto moderadamente positivo de sí mismos.

Al comparar el autoconcepto de niños/as con y sin DPS no se encuentran diferencias significativas, a excepción del autoconcepto físico en el que los/as niños/as con DPS obtuvieron puntajes superiores, sugiriendo que estos/as niños/as presentan una mejor percepción de sí mismos/as, en comparación con los/as niños/as sin DPS. Estos resultados no guardan relación con lo propuesto por autores como Ayres (1998); Parhan \& MaiIlloux, (2001); Bundy et al., (2002) quienes plantean que el autoconcepto, el autocontrol y la confianza en sí mismo se ven afectadas en los niños/as con DPS, así como el estudio de Brown \& Carney (2020) que sostiene que el autoconcepto se ve afectado en niños con problemas de coordinación motora, o el estudio de Maïano, Coutu, Morin, Tracey, Lepage \& Moullec (2019) que plantean el impacto negativo en el autoconcepto en adolescentes con discapacidad cognitiva, ya que estos sentimientos

TABLA 3. PRUEBA T DE STUDENT PARA LAS DIMENSIONES DE AUTOCONCEPTO

\begin{tabular}{|c|c|c|c|c|c|c|}
\hline & \multicolumn{2}{|c|}{$\begin{array}{l}\text { Grupo sin DPS } \\
\quad(n=60)\end{array}$} & \multicolumn{2}{|c|}{$\begin{array}{c}\text { Grupo con DPS } \\
(n=38)\end{array}$} & \multirow[t]{2}{*}{$t(g \mid)$} & \multirow[t]{2}{*}{ Sig } \\
\hline & Media & DT & Media & DT & & \\
\hline Autoconcepto conductual & 15.62 & 3.031 & 15,43 & 2,398 & $0.31(95)$ & 0.754 \\
\hline Autoconcepto social & 12.17 & 2.345 & 12.39 & 2.138 & $0.485(96)$ & 0.629 \\
\hline Autoconcepto emocional & 8.15 & 2.523 & 8.87 & 2.896 & $1.296(96)$ & 0.198 \\
\hline Autoconcepto felicidad & 6.60 & 2.093 & 9.18 & 1.608 & $1.467(96)$ & 0.146 \\
\hline Autoconcepto físico & 4.52 & 1.321 & 5.18 & 1.353 & $2.415(96)$ & $0.018^{*}$ \\
\hline Autoconcepto general & 8.13 & 1.200 & 7.82 & 1.312 & $1.231(96)$ & 0.221 \\
\hline
\end{tabular}

*significativo al 0,05 
provienen de sentir que el cuerpo es un ente sensoriomotor competente, lo cual requiere como base una buena integración neurológica. Posiblemente la diferencia en los resultados obtenidos se debe a que los niños/as con DPS se encontraban en procesos terapéuticos, por tanto, sus fallas en el procesamiento sensorial han sido abordadas. En este sentido, probablemente tuvieron una constante retroalimentación positiva por parte de terapeutas y padres, quienes conocían la razón de sus dificultades y podían resaltar sus características físicas para quitarle fuerza a la percepción de sus dificultades a nivel funcional, colocando a los/as niños/as con DPS en ventaja frente a los/as niños/as sin déficit. Otro aspecto que posiblemente puede guardar relación con los resultados encontrados es la edad de los participantes, ya que los/as niños/as sin DPs tenían una edad media mayor $(10,3)$ que los/as niños/as con DPS $(8,8)$, ya que como plantean Soriano, et al. (2014) y Nobre \& Valentini, (2019). el autoconcepto físico puede ir sufriendo cambios a lo largo de los años debido a las diversas experiencias que la persona enfrenta, mostrando un descenso principalmente en la entrada de la adolescencia que luego es solventado cuando la persona se adapta a las transformaciones, momento en el que el autoconcepto aumenta nuevamente.

En cuanto a la variable autoeficacia, al comparar los resultados entre los dos grupos, se encuentra que tanto en la dimensión académica como lúdico social, los/ as niños/as con DPS obtienen resultados más bajos que

TABLA 4 PRUEBA T DE STUDENT PARA LAS DIMENSIONES DE AUTOEFICACIA

\begin{tabular}{lllllll}
\hline & \multicolumn{2}{c}{$\begin{array}{c}\text { Grupo sin DPS } \\
(\mathbf{n = 6 0})\end{array}$} & $\begin{array}{c}\text { Grupo con DPS } \\
(\mathbf{n = 3 8 )}\end{array}$ & t(gl) & Sig \\
\cline { 1 - 5 } & Media & DT & Media & DT & & \\
Autoeficacia académica & 66.07 & 8.747 & 62.00 & 10.516 & $2.702(96)$ & $\mathbf{0 . 0 4 1}^{*}$ \\
Autoeficacia lùdico-social & 47.42 & 5.918 & 43.58 & 7.504 & $2.815(96)$ & $\mathbf{0 . 0 6}^{*}$ \\
\hline
\end{tabular}

*significativo al 0,05

TABLA 5 DIMENSIONES POR CALIDAD DE VIDA POR GRUPO. PRUEBA T DE STUDENT

\begin{tabular}{|c|c|c|c|c|c|c|}
\hline & \multicolumn{2}{|c|}{$\begin{array}{l}\text { Grupo sin DPS } \\
(n=60)\end{array}$} & \multicolumn{2}{|c|}{$\begin{array}{l}\text { Grupo con DPS } \\
(n=38)\end{array}$} & \multirow[t]{2}{*}{$\mathbf{t}(\mathrm{gl})$} & \multirow[t]{2}{*}{ Sig } \\
\hline & Media & DT & Media & DT & & \\
\hline Bienestar físico & 20.88 & 3.068 & 20.29 & 3.229 & $0.86(99)$ & 0.411 \\
\hline Bienestar psicológico & 26.90 & 3.602 & 26.68 & 2.440 & $0.353(95.51)$ & 0.725 \\
\hline Estado de ánimo & 27.73 & 6.709 & 28.68 & 4.563 & $0.835(95.46)$ & 0.406 \\
\hline Autopercepción & 22.02 & 3.666 & 21.84 & 3.089 & $0.244(96)$ & 0.808 \\
\hline Autonomía & 19.60 & 4.872 & 20.97 & 3.027 & $1.722(95.98)$ & 0.880 \\
\hline Relación con los padres & 26.17 & 4.361 & 26.21 & 3.314 & $0.053(96)$ & 0.958 \\
\hline Recursos económicos & 10.62 & 3.880 & 9.89 & 3.494 & $-0.932(96)$ & 0.354 \\
\hline Amigos y apoyo social & 25.98 & 5.525 & 25.87 & 2.820 & $-0.136(92.55)$ & 0.892 \\
\hline Entorno escolar & 24.13 & 6.560 & 25.76 & 3.373 & $1.617(92.76)$ & 0.109 \\
\hline Aceptación social & 11.98 & 3.557 & 12.79 & 2.055 & $1.415(95.26)$ & 0.160 \\
\hline
\end{tabular}

*significativo al 0,05 
los/as niños/as sin DPS, sin embargo, solo hay diferencia significativa para la dimensión Autoeficacia Académica. Teniendo en cuenta que los/as niños/as con DPS presentan dificultades en su desempeño académico (Ayres, 1998, Smith, 2006, Schaaf, \& Mailloux, 2015), este resultado apoya lo planteado por Medeiros, et al. (2000), quienes observaron que los/as niños/as con rendimiento académico bajo, muestran un sentido de autoeficacia significativamente menor que los/ as niños/as con buen rendimiento académico, al igual que lo planteado Stajkovic, A.D. Bandura, A., Locke, E., Lee, D. \& Sergent, K. (2018) y Wen- hong, \& Yi (2019), en adolescentes. Ambos estudios concluyeron entre otras cosas, que el sentido de autoeficacia se relaciona con el rendimiento académico.

Así mismo, los resultados obtenidos al evaluar la autoeficacia coinciden con los resultados dados por Cairney, et al. (2005), quienes estudiaron la autoeficacia para la actividad física y la participación en actividades de juego de niños/as canadienses con DPS, específicamente con desorden del desarrollo de la coordinación y encontraron que estos/as niños/as poseen una baja autoeficacia generalizada y tienden a participar menos en actividades físicas y en el juego.

Finalmente, al comparar la calidad de vida de niños/as con y sin DPS no se encontraron diferencias significativas en ninguna de las dimensiones; difiriendo de los obtenidos por Karras et al,. (2017), quienes al estudiar la Calidad de Vida en niños/as con DPS, específicamente con Desorden del Desarrollo de la Coordinación, encontraron que estos/as niños/as presentan menor calidad de vida, así mismo, plantean que las dificultades en el desempeño motor, los cuales se presentan comúnmente en los DPS, predicen la calidad de vida. Igualmente, contradice el estudio de Mensch, EchteId, Lemmens, Oppewal, Evenhuis, \& Rameckers (2019) quienes encontraron que los niños con mejor rendimento motor presentaban mejor calidad de vida.

De igual manera, autores como Verdugo \& Sabeh (2002), Klassen, Miller \& Fine (2004) y Miranda-Casas, et al. (2011), estudiaron la calidad de vida en niños/as con condiciones que afectan el desempeño ocupacional y/o la participación como dificultades de aprendizaje y trastorno de déficit de atención e hiperactividad. Los autores mencionados concluyeron en sus investigaciones que los/as niños/as con estas condiciones tienen una calidad de vida más baja que el resto de sus compañeros y que estas condiciones tienen un impacto significativo en términos de menor autoestima, habilidades emocionales deficientes y pobres relaciones sociales.

Así mismo, López-Villalobos et al. (2018), estudiaron la calidad de vida en niños/as con trastorno por déficit de atención/hiperactividad (TDAH) con y sin tratamiento farmacológico y en controles, mediante el uso del instrumento KIDSCREEN -52, encontrando una correlación significativa moderada entre mayor intensidad de síntomas de TDAH y peor calidad de vida, especialmente en las dimensiones bienestar psíquico, autonomía, estado de ánimo, entorno escolar y aceptación social.

Por su parte, Cairney, et al. (2005), aunque no evalúan directamente esta variable, plantean la importancia de tenerla en cuenta en las investigaciones con niños/as con DPS, resaltando que es fundamental el desarrollo de intervenciones psicosociales dirigidas a mejorar la supervivencia y la calidad de vida de estos/as niños/as.

\section{LIMITACIONES}

Respecto a las limitaciones del presente estudio se puede resaltar que, aunque el tamaño de la muestra era suficiente, sería ideal que el número de sujetos en cada grupo fuera equivalente, aspecto que no fue posible debido a la dificultad para acceder a niños/as con DPS sin ningún diagnóstico asociado. Así mismo, debe asegurarse el control de la variable edad entre los grupos, para de esta manera asegurar que las diferencias encontradas no son debidas al crecimiento sino a diferencias relacionadas con el DPS. Por otro lado, el hecho de no contar con suficiente evidencia que relacione el DPS con variables psicológicas, limitó la posibilidad de hacer comparaciones con estudios previos, siendo necesario valerse de estudios realizados en niños/as con otras condiciones, que, si bien afectan su desempeño y participación al igual que en el caso del DPS, no era específicamente la población estudiada en el presente estudio. Finalmente, se considera que el hecho de que el grupo de niños/as con DPS se encontrará en procesos terapéuticos pudo tener un impacto sobre resultados, por lo que se considera que en futuros estudios sería ideal contar con un grupo de niños/as con DPS sin intervención, uno con intervención terapéutica y el grupo control. 


\section{ConCLUSIÓN}

Esta investigación permitió abrir un camino en el estudio de variables psicológicas que han sido poco estudiadas y abordadas en niños/as con DPS, demostrando que esta condición puede impactar de manera negativa la percepción que tiene el/la niño/a de sus habilidades y autoconcepto. El procesamiento sensorial es considerado como la base para el desarrollo de los diferentes componentes del desempeño, por tanto, un DSP puede ocasionar dificultades en la ejecución de tareas cotidianas que parecen sencillas, lo que puede influenciar negativamente en la creencia de las propias habilidades y consecuente en su percepción de autoeficacia y su autoconcepto.

Los resultados son considerados un aporte tanto para la práctica como para la investigación, ya que marcan el camino de inicio en el estudio de una población que actualmente muestra niveles crecientes de prevalencia y que ha sido muy poco estudiada en Venezuela, lo cual impactará positivamente la práctica profesional ya que, posiblemente al abordar estas variables psicológicas durante la intervención, los resultados en términos de desempeño ocupacional y bienestar podrían verse beneficiados. A su vez, la intervención de integración sensorial tiene un impacto positivo en los diferentes componentes del desempeño, incluyendo el socioemocional (Ayres 1998; Schaaf \& Smith, 2006; Schaaf \& Mailloux, 2015, Blanche, Chang, Gutiérrez \& Gunter, 2016).

\section{AGRADECIMIENTOS}

Las autoras agradecen a la Coordinación de la Maestría en Psicología de la Universidad Simón Bolívar y su cuerpo profesoral, a la Escuela de Salud Pública de la Universidad Central de Venezuela, a las instituciones educativas, padres y profesores que apoyaron la investigación y en especial a los niños y niñas que hicieron parte de la muestra de estudio.

\section{RefERENCIAS BibLIOGRÁfiCAS}

Ato, M. \& Vallejo, G. (2015). Diseños de investigación en Psicología. Madrid: Ediciones Pirámide.

American Occupational Therapy Association (2014). Research Opportunities in the Area of Children and Adolescents with Challenges in Sensory Processing and Sensory Integration. American Journal of Occupational Therapy, 68, 242-244. https://doi.org/10.5014/ ajot.2014.682003

Ayres, J. (1998). La integración sensorial y el Niño. México: Editorial Trillas. Bandura, A. (1990). Multidimensional scales of perceived self-efficacy: California: Standford University.

Blanche, E., Chang, M., Gutiérrez, J., \& Gunter, J. (2016). Effectiveness of a Sensory-Enriched Early Intervention Group Program for Children with Developmental Disabilities. American Journal of Occupational Therapy, 70 (5), 1 - 8. https://doi.org/10.5014/ ajot.2016.018481

Brown DMY, Cairney J. (2020) The synergistic effect of poor motor coordination, gender and age on self-concept in children: A longitudinal analysis. Reseatch in Development Disabilities, 98:103576. https://doi.org/10.1016/j.ridd.2020.103576

Bundy, A., Lane, S. \& Murray, E. (2002). Sensory Integration. Theory

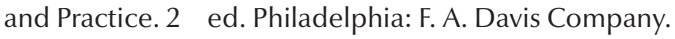

Caçola, P., \& Killian, M. (2018). Health-related quality of life in children with Developmental Coordination Disorder: Association between the PedsQL and KIDSCREEN

Cairney, J., Hay, J., Faught, B., Wade, T., Corna, L., \& Flouris, A. (2005). Developmental coordination disorder, generalized self-efficacy toward physical activity, and participation in organized and free play activities. Toronto: J Pediatr, 147 (4), 515-20.

Carrasco, M., \& Del Barrio, V. (2002). Evaluación de la Autoeficacia en niños y adolescentes. Psicothema, 14 (2), 323-332. Recuperado de http://www.psicothema.com/psicothema.asp?id=727

Colombo, M. (2003). Integración sensorial y distintos estilos de desarrollo del niño. Recuperado de http://ceril.cl/index.php/ profesionales-2/12-publicaciones/articulos/52-integracion-sensorial-y-distintos-estilos-ceril

Dunn, W. (1999). Sensory Profile, User's Manual. USA: The Psychological Corporation.

Fisher, A., Murray, E., \& Bundy, A. (1991). Sensory Integration, Theory and Practice. Philadelphia: F. A. Davis Company.

Guzmán, N. (2012). Validación de los Cuestionarios de Calidad de Vida KIDSCREEN y DISABKIDS en niños y adolescentes venezolanos. (Tesis Doctoral). Universidad de Alicante. Alicante, España.

Hernández, R, Fernández, C., \& Baptista, M.P. (2014). Metodología de la Investigación. 6ta ed. México: McGraw Hill Education.

Karras, H. C., Morin, D. N., Gill, K., Izadi-Najafabadi, S., \& Zwicker, J. G. (2019). Health-related quality of life of children with Developmental Coordination Disorder. Research in developmental disabilities, 84, 85-95. https://doi.org/10.1016/j.ridd.2018.05.012

Klassen, A., Miller, A. \& Fine, S. (2004). Health-related quality of life in children and adolescents who have a diagnosis of attentiondeficit/hyperactivity disorder. Pediatrics, 114 (5), e541 -e547.

Kumar, R. (2011). Research Methodology. A step-by-step guide for beginners. 3er Ed. London: SAGE publication Ltd. 
López-Villalobos, JA., Garrido Redondo, M., Sacristán-Martín, AM., Martínez-Rivera, MT., López-Sánchez, MV., Andrés-De Llano, JM., Rodríguez-Molinero, L., Camina-Gutiérrez, A. (2018). Percepción de niños y adolescentes sobre la calidad de vida en casos de trastorno por déficit de atención/ hiperactividad con y sin tratamiento farmacológico y en controles. Rev Neurol, 67, 195-202.

Maïano, C, Coutu, S, Morin, AJS, Tracey, D, Lepage, G, Moullec, G. (2019). Self-concept research with school-aged youth with intellectual disabilities: A systematic review. Journal of applied Resesarch Intellectaul Disabilities, + 32: 238-255. https://doi. org/10.1111/jar.12543

Medeiros, P., Loureiro, S., Linhares, M. \& Marturano, E. (2000). La autoeficacia y los aspectos conductuales de los niños con dificultades de aprendizaje. Psicología: Reflexao e Crítica. 13(3), 227-336.

Mensch, S. M., Echteld, M. A., Lemmens, R., Oppewal, A., Evenhuis, H. M., \& Rameckers, E. A. A. (2019). The relationship between motor abilities and quality of life in children with severe multiple disabilities. Journal of Intellectual Disability Research, 63(2), 100-112. https://doi.org/10.1111/jir.12546

Miranda-Casas A, Presentación- Herrero M, Colomer-Diago C \& Roselló B, (2011). Satisfacción con la vida de niños con trastorno por déficit de atención/hiperactividad: estudio de posibles factores de riesgo y de protección. Revista Neurología 52 (1), 119-26.

Nobre, G.C. \& Valentini, N.C. (2019). Self-perception of competence: concept, changes in childhood and gender and age-group differences. Journal of Physical Education, 30, e3008. https://dx.doi. org/10.4025/jphyseduc.v30i1.3008

Parham, D. \& Mailloux, Z., (2001). Sensory Integration. In Jane CaseSmith (Coord.), Occupational Therapy for Children. (pp. 329 -279). St. Louis, Missouri: Weastline Industrial Drive.

Pfeiffer, B., Coster, W., Snethen, G., Derstine, M., Piller, A., \& Tucker, C. (2017). Caregivers' perspectives on the sensory environment and participation in daily activities of children with autism spectrum disorder. American Journal of Occupational Therapy, 71, 7104220020. https://doi.org/10.5014/ajot.2017.021360

Piers, E. \& Harris D. (1969). The Piers-Harris Children's Self-Concept Scale. Nashville, Tennesse: Counselor recording and Tests.

Plata, R. \& Garbiñe, B. (2009). El niño con trastorno del desarrollo de la coordinación $i U n$ desconocido en nuestra comunidad? Revista NORTE de Salud Mental. 33, 18-30.

Poulsen, A. \& Ziviani, J. (2004). Can I play too? Physical activity engagement patterns of children with developmental coordination disorders. Canadian Journal of Occupational Therapy, 71 (2), 100-107.

Ravens-Sieberer, U., Erhardt, M., Wille, N., Wetzel, R., Nickel, J. \& Bullinger, M. (2006). Gemeric health-related qualite-of-life assessment in children and adolescents: methodological considerations. Pharmacoecomomics, 24 (12), 1199-220.

Serrano, P. (2019). La Integración Sensorial: en el desarrollo y aprendizaje infantil. Madrid: Narcea Ediciones.
Schaaf, R., \& Smith Roley, S. (2006). Sensory Integration: Applying Clinical Reasoning to Practice with Diverse Populations. Texas: Pro-Ed.

Schaaf, R., \& Mailloux, Z. (2015). Clinician's Guide for Implementing Ayres Sensory Integration: Promoting Participation for Children with Autism. USA: American Occupational Therapy Association, Inc.

Smith Roley, S. (2006). Sensory Integration Theory Revisited. In Schaaf, R., \& Smith Roley, S. (Coord.), Sensory Integration: Applying Clinical Reasoning to Practice with Diverse Populations (pp. 1 - 13). Texas: Pro-Ed.

Soriano, J., Navas, L. \& Holgado, F. (2014). El autoconcepto físico y su relación con el género y la edad en estudiantes de educación física. Apunts Educación física y deportes, No.106, 36-41.

Stajkovic, A.D., Bandura, A., Locke, E., Lee, D. \& Sergent, K. (2018). Test of three conceptual models of influence of the big five personality traits and self-efficacy on academic performance: A meta-analytic path-analysis. Personality \& Individual Differences 120, 238-245. https:// Doi10.1016/j.paid.2017.08.014.

Wen- hong Sun, \& Yi- Jian Huang. (2019). Research on Relationships among Achievement Goals, Self- Efficacy and Academic Performance of Students in Shandong Province. International Journal of Organizational Innovation, 12(1), 280-289.

Verdugo, M. Á. \& Sabeh, (2002). Evaluación de la percepción de calidad de vida en la infancia [Evaluación de la calidad de vida percibida en la infancia]. Psicothema, 14 (1), 86-91. 